Revue d'histoire de l'Amérique française

PEV RUE D.HISTOIRE DE L'AMÉRIQUE FRANÇAISE

\title{
Un ancien registre paroissial ontarien
}

\section{George F. G. Stanley}

Volume 8, numéro 3, décembre 1954

URI : https://id.erudit.org/iderudit/301668ar

DOI : https://doi.org/10.7202/301668ar

Aller au sommaire du numéro

Éditeur(s)

Institut d'histoire de l'Amérique française

ISSN

0035-2357 (imprimé)

1492-1383 (numérique)

Découvrir la revue

Citer ce document

Stanley, G. F. G. (1954). Un ancien registre paroissial ontarien. Revue d'histoire de l'Amérique française, 8(3), 426-434. https://doi.org/10.7202/301668ar d'utilisation que vous pouvez consulter en ligne.

https://apropos.erudit.org/fr/usagers/politique-dutilisation/ 


\section{DOCUMENTS INEDITS}

\section{UN ANCIEN REGISTRE PAROISSIAL ONTARIEN}

Le 12 juillet 1673, Louis de Buade, Comte de Frontenac et gouverneur de la Nouvelle-France, et Robert Cavelier, Sieur de la Salle, débarquèrent sur la côte nord du lac Ontario à l'endroit où se trouve aujourd'hui la ville ontarienne de Kingston. Sur les ordres de Frontenac, l'ingénieur Randin y construisit une palissade. ' C'était une fortification rudimentaire, le premier de plusieurs forts érigés sur le même site. ${ }^{2}$ En 1689 le gouverneur Denonville abandonna ce petit fort: mais Frontenac le rétablit à son retour au Canada comme gouverneur pour la deuxième fois. Au cours des années qui suivirent le Fort Frontenac (ou Cataracoui) remplit à la fois le rôle de comptoir de traite et de fortification militaire. Situé à la source du fleuve St. Laurent et à l'embouchure de la rivière Cataracoui il avait de grands avantages aussi bien pour les trafiquants que pour les soldats. En août 1756 il servit de base d'opérations pour la marche contre Chouagen (Oswego). Deux ans plus tard le commandant, Pierre-Jacques Payan, Sieur de Noyan, le rendit au Colonel Bradstreet. Ce dernier n'y resta pas; après avoir détruit une grande partie des fortifications, il retourna avec ses troupes aux colonies anglo-américaines. L'ingénieur Pontleroy fit un examen sommaire des ruines et conseilla à ses supérieurs de ne pas le rebâtir; quelque temps après le Chevalier de Lévis fit également une reconnaissance du fort mais sans le réoccuper. Le rêve de Frontenac et de la Salle d'un empire occidental s'écroulait; d'autres allaient le faire revivre.

1. Voir à ce sujet Antoine Roy, Le Fort Frontenac (ou Catarakoui) sous le régime franşais (Rapport annuel de la société bistorique du Canada, 1950); Benjamin Sulte, Le Fort Frontenac (Mémoires de la société royale du Canada, 1901); Pierre Margry, Mémoires et documents (Paris, 1874-88) 1 : 198.

2. Le collège d'état-major de l'armée canadienne, qui perpétue le nom du Fort Frontenac, occupe aujourd'hui le site des anciens forts français. 
Pendant ces jours héroïques de l'ancien régime le bien-être spirituel fut confié aux soins des pères Recollets. On érigea une chapelle en dehors des murs et c'est là qu'on disait la messe pour les troupes de la garnison, pour les habitants du voisinage, et pour les sauvages. On ignore presque tout de la vie des gens du fort et de celle des missionnaires; heureusement on peut recueillir quelques renseignements dans le Registre des Baptesmes, Mariages et Sepultures de la Paroisse de St. François, document qui se trouve actuellement dans les archives de l'église de Notre Dame à Montréal. ${ }^{3}$ Le Registre contient les actes des baptêmes, des mariages et des sépultures des Français et des sauvages chrétiens au Fort Frontenac, de novembre 1747 à mai 1752. Ces actes sont de la main des aumôniers CharlesAntoine-Isidore Lemire-Marsolet, Louis-Marie-Bonaventure Carpentier, et Nicolas-Albert Couturier. Le Registre révèle l'existence de différends entre le commandant, le Sieur de Vassan, et le Père Couturier, au sujet des heures de la messe, de l'emploi de la cloche de la chapelle, et de certains hommages personnels que réclamait le commandant à l'église.

Bien que plusieurs écrivains aient consulté le Registre, ${ }^{4}$ ce document est peu familier aux historiens canadiens. Il est certain que le Registre n'est pas très important au point de vue de l'histoire du Canada; sa signification est plutôt locale que générale. Mais il mérite l'attention, quand ce ne serait que pour jeter un peu de lumière sur les premiers jours d'un établissement franco-ontarien.

George F. G. Stanley, Kingston, Ontario.

3. Une copie de l'original de ce document a été déposée aux archives de la province d'Ontario à Toronto. - La Revue offre ici à ses lecteurs une copie colllationnée sur l'original déposé aux archives de l'église de Notre-Dame de Montréal. (La Direction).

4. Notamment James Roy, Kingston, the King's Town (Toronto, 1952) 9-10; et Léopold Lamontagne, Kingston's French Heritage (Ontario History, summer 1953) 110. 
R E G I S T R E

des

\section{BAPTESMES, MARIAGES ET SEPULTURES}

de la paroisse de St-françois

fort Royal: De frontenac

Commencé

Le 10e 9bre 1747

\section{7}

* 1. L'an mil sept Cent quarante sept, Le dixiesme novembre par moy pretre Recollet aumonier du Roy a Etté inhumé dans Le Cimetiere de ce poste, Le fils d'un missisagué nommé La Croupe; agé d'environ six ans, Lequel a Etté Baptisé par $\mathrm{m}^{\mathrm{r}}$. piquet ce printemps, ont Ettés present a son inhumation messieurs St. paul Senneville et du Chouquet Et plusieurs autres, En foy de quoy Jay signé Les Jours Et ans que dessus

$$
\begin{array}{r}
\text { fr. Isidore Marsollet } \\
\mathrm{R} \text { aumonier }
\end{array}
$$

\section{8}

2. Lan mil sept cent quarante huit, Le Dix huitiesme febvrier par moy pretre Recollet aumonier du Roy au fort frontenac a Ettée baptisé une fille agée Environ de deux mois nommée marie Catherine fille dun huron du Detroit Et dune femme iroquoise du Lac; Le parain a Etté Jean baptiste Ecuyer Sieur de St paul Senneville, officier dans Cette garnison, Et La maraine damoiselle marie Catherine Duchouquet, Lesquels ont signé avec moy les jours \& ans que Dessus

St. paul Senneville catberine ducbouquet fr. Isidore marsollet

$R$.

\footnotetext{
* Ce numérotage des actes du Registre a été fait à la mine sur le $M_{s}$ et les chiffres sont à la marge.
} 


\section{8}

3. Lan mil sept Cent quarante huit, Le dixseptieme doctobre par moy pretre Recollet a Etté inhumée dans le Cimetiere de ce poste marie anne fille de Chistaron iroquois du Lac, agée Denviron de huit a neuf ans, morte de puis quatre a Cinq Jours, ont Etté presents a son inhumation $\mathrm{m}^{\mathrm{r}}$. de Lavalliere, La forge Et plusieurs autres, en foy de quoy, jay signé Les jours Et ans que Dessus

$$
\begin{array}{rlr} 
& f r . \text { Isidore marsollet } R \\
1748 & \text { aumonier }
\end{array}
$$

4. Lan mil sept quarante huit Le vingt Cinq doctobre par moy pretre Recollet a Etté inhumé dans Le Cimetiere de Ce poste Le corps de marie Joseph fille de Chistoron iroquois du Lac, decedee dhier agée Environ de deux ans, Ont Ettés presents a son inhumation messieurs de Lavallieres Et plusieurs autres, En foy de quoy Jay signé les jour Et an que dessus

$$
\begin{array}{rlrl} 
& & \text { fr. Isidore marsollet } R \\
& & & \text { aumonier }
\end{array}
$$

5. Lan mil sept cent quarante huit Le vingt de decembre par moy aumonier du Roy au fort frontenac a Ettée Baptisée Elizabeht fille de Joseph Levron dit metayer Et de marie Joseph Custeau ses pere Et mere; nee de ce jour Le parain a Etté Jean Baptiste Ecuyer sieur De vassant Commendant pour le Roy dans ce poste Et La mareinne Elizabeht Lemire femme du sieur Du Chouquet les quels ont signés auec moy les jours Et an que Dessus

$$
\text { Devassan }
$$$$
\text { elissabete lemire }
$$

\section{9}

fr. Isidore marsollet

6. Lan mil sept cent quarante neuf Le sept de febvrier, par moy pretre Recollet missionnaire au fort frontenac, a Ettée inhumée dans le Cimetiere de ce poste marie anne fille de glaude tegat scaranet et de susane teya8evinhon iroquois du Lac, décédée depuis un mois agée d'environ quatre ans, ont Ettés presents a son inhumation messieurs Lavalliere du Chouquet Laforge Et plusieurs autres, En foy de quoy Jay signé Les jours Et an que dessus.

\section{fr. Isidore marsollet}

$\mathbf{R}$ aumonier 


\section{9}

7. Lan mil sept Cent quarante neuf Le huitiesme de febvrier par moy pretre Recollet missionnaire au fort frontenac a Ettee bapptisé selon les Ceremonies de notre mere la sainte Eglise, therese gatsiragueron fille de jaque gatsirageron Et de margueritte ses pere et mere iroquois du sault St Louis; Elle Etoit agee denviron un mois, Le parain a Ette Joseph boisneuf de Lavalliere officier des troupes de Lisle Royalle Et la mareinne demoiselle therese lefebvre $\mathrm{du}$ chouquet Lesquels ont signe avec moy Les jour et an que dessus

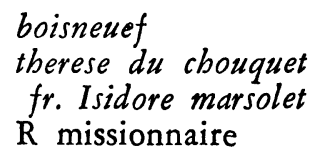

8. Lan mil sept Cent quarante neuf le premier mars par moy pretre Recollet missionnaire au fort frontenac a Ette inhumée dans le Cimetiere de cette paroisse le Corps de Cecile, fille de Sapitié argontien du Lac, décédée du sixiesme janvier, ont Etté presents a son inhumation messieurs becancourt Du chouquet Et plusieurs autres, En foy de quoy Jay signé les jour et an que dessus

fr. Isidore marsollet

$\boldsymbol{R}$.

9. Lan mil sept Cent quarante neuf Le dixseptiesme Juin par moy pretre Recollet a Etté Baptisé un garcon nommé nicolas agé denviron neuf mois, fils de margueritte Sotacoan iroquoise du Lac, le pere est inconnû Le parain a Etté nicolas Catin fils et la mareinne Elisabeht La favry, Lesquels ont de claré ne scavoir Ecrire ny signer, En foy de quoy Jay signé Les jour Et an que dessus.

fr. Isidore marsollet

$R$.

\section{0}

Lan mille sept cent cinquante le 26 Jours deJanvier apres avoir publié un banc selon $\mathrm{La}$ permission anous donnée par monseigneur entre joseph Levron veuf de rose denis veronno, fils de Pierre Levron et dagnes testu ses pere et mere natif de $\mathrm{La}$ cadis dune part et Catherine brunet veuve de daniel fille de feu mathieu brunet et de 
marie blanchar ses pere et mere de la paroisse de montreal residans au fort frontenac sans quil y ait eu aucun empeschement Je sousigné missionnaire du fort frontenac ait recu leur mutuel consentement de mariage et Leur ai donné La benediction nuptiale avec Les Ceremonies prescrites par la $\mathrm{S}^{\text {te }}$ eglise en presence des temoins qui ont signé avec moi de ce interpellé suivant lordonnance

\section{Duchouquet \\ Lefebure \\ Pierre Baron \\ f. bonnavanture}

Lan mille sept cent cinquante Le 6 davril apres avoir publié deux bans entre francois billard.

11. Reglement fait par monseigneur approuvé par $\mathrm{m}^{\text {re. }}$ Le general premierement que le missionaire nest point obligé daller chaque jours prendre Lheur de lamesse ni dattendre la reponse. 2 . cette heur etant une fois marquée ce sera a $\mathrm{m}^{\text {re. }}$ le commendant de faire avertir Laumonier quil est necessaire pour lebien du service davancer ou de retarder et il convient alors que Laumonier suivent ses ordres. 3. que Laumonier habillé passant devant Luy, n'est point obligé de Luy faire une inclination. 4. il n'est point du au commendant Lencen ni Leaubenite. $\mathbf{M}^{\text {re. }}$ Lintendant napas ses deux premieres marques d'honneur et ce nest qu'apres bien des difficultes qu'on Les a accordées $a^{\text {re. }}$ Le general. 5. que la chose etant benite a Lusage dela chapelle qui en est en possession depuis pres de vingttemps nesera employé adautres usages.

Pour copie du Reglement fait par Mgr. De Pontbriand Evesque de Quebec ce 26 Mars 1750 Quod Testor hac die die 28a novembris Ejusdem anni $I$. Nicol. Alb. Couturier

\section{0}

12. Lan mille sept cent cinquante le 6 davril apres avoir publié deux bans entre francois billard natif de la paroisse St. germain evesché de charte ambos fils de feux francois billard et de marguerite Lorin ses pere et mere d'une part et marie demoulin fille de feux Louis Joseph demoulin et de marianne grandbois ses pere et mere dautre part residans au fort frontenac sans quil y ait eu aucun empeschement Je sousigné missionnaire dudit fort avoir receu Leur mutuel consentement demariage et Leur ai donné Labene- 
diction nuptialle avec les ceremonies prescrites par la Ste eglise en presence des temoins sousignés de ce requis Lecture faite suivant Lordonnance

Devassan
Berey devassan
Jacquespée
François Billard
Duvergé St blin
Duchouquet
Chevalier De Portneuf
Lorimier Jacques
f. bonnavanture
Carpentier

marie demoulin adeclaré ne scavoir signee elle a fait sa marque ordinaire +

\section{1}

13. Ce cinqe. de Lan 1751 mille Sept Cent Cinquante et un a Eté Inhumé dans le Cimetiere de cette Garnison Le Corps de petit Jean Batiste Levron dit metayer fils de Joseph Levron Metayer et de Marie Joseph Custeau ses pere et mere; Led. Enfant de. [décédé] cette nuit Vers les douze heures, agé de trois ans. En foy de quoy Les personnes soussignées ont auec nous Signé le present acte

\section{Du Chouquet}

Vancauret

J. Nicolas albert Couturier

14. Ce douzieme avril 1751, a eté Batisée Elizabeth Catherine née depuis vingt jours fille de Pierre Kaüighin8achyé et de Marie Kanuoinsté du Sault St Louis. le parein aeté Thomas Chiratongouté, et la Maraine Mere (mot illisible) Duchoquet laquelle sceu signer

\section{elissasbetelemire \\ I. nicolas albert.Couturier}

\section{1}

15. Sepult. de francois Poisson. Ce Dix avril mil sept cent Cinquante un a eté Inhumé dans Le Cimetiere francois Poisson mort dhier apres midy, agé de soixante et trois ans, apres avoir reçue Les sacrements, En foy de quoy nous avons Signé Le Sr. Duchouquet et moy

Duchouquet

I. nicolas albert Couturier 
16. Bapt. D'Elizabeth Charly Rénard. Ce Sept May 1751 a eté Batisée Elizabeth née Le Cinq de Decembre de lannée precedente, fille de Jean Baptiste Charly Rénard, et de Marie Anne Koüilloucénde Ses pere et Mere en Legitime mariage habitans du lac des montagnes. Le Parain a eté Sieur charles De Vaucoret la Maraine Elizabeth Lafavry, Lesquels pour foy du present acte ont Signé avec nous Les Jour et an que dessus

$$
\begin{aligned}
& \text { Jean C. Vaucauret } \\
& \text { elisab. Lafavry } \\
& \text { J. nicolas albert Couturier }
\end{aligned}
$$

17. Sepult. De Joseph La Beaume. Ce trente de juin de l'année mil sept Cent Cinquante et vn a été Inhumé dans le Cimetiere Le Corps de feu Joseph La Beaume Né a grenoble au village de Moretel agé d'environ quarante ans, engagé dans les trouppes de ce Païs, Mort de cette Nuit vers vne heure, Muni de ses Sacrements en foy de quoy nous avons signé les jours et an que Dessus

\section{J. Nicolas Albert Couturier}

18. Ce dix huit Juillet 1751 apres la publication des Bans de Mariage Entre Joseph Guibeau fils de Jean Guibeau \& de Marie Vien, natif de Ste. anne, et Angelique De Berey de nation Siouze; ne S'etant trouvé aucun Empeschement Je Soussigné Aumônier du fort frontenac Leur ay donné La Benediction nuptiale presence des Temoins soussignés.

$$
\begin{aligned}
& \text { Mutigny de Vassan } \\
& \text { Du Chouquet } \\
& \text { Malbiot } f \text {. } \\
& \text { Lantagnac } \\
& \text { J. Nicolas Albert Couturier }
\end{aligned}
$$

\section{2}

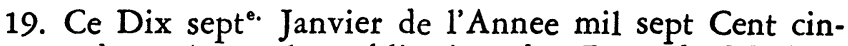
quante deux Apres la publication des Bans de Mariage entre joseph Mercier fils d'Antoine Mercier et de Claudine Millet de la Paroisse de St. Augustin de Quebec ses pere et Mere, agé de vingt six Ans d'une part; Et Marie Joseph Levron, fille de Sieur Joseph Levron dit Metayer Capitaine des Batimens du Roy sur le lac ontario et de feüe Rose Denys Ses pere et mere âgée d'environ de dix sept ans d'autre part; ne s'etant trouvé aucun empeschement, Je sous- 
signé aumonier du fort frontenac ay reçu a la Ste Messe leur consentement mutuel, et leur ay donné la Benediction nuptiale presence des Temoins Sous Signés.

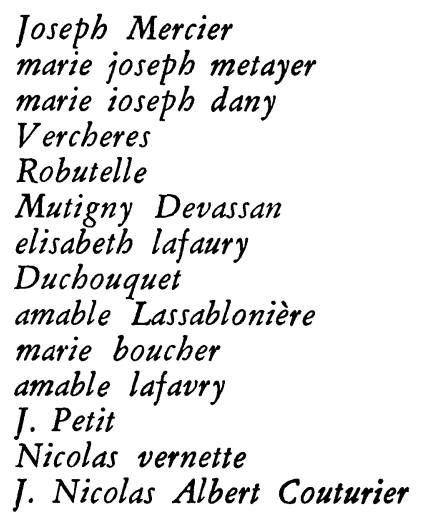

20. Sepult. de Louis. Ce huit Mars 1752 a été Inhumé dans le Cimetiere Le Corps de Loùis Le Castor noir Mississagué que J'avois Batisé Cinq a six jours avant sa mort; lequel etoit agé d'environ vingt ans; En foy de quoy Jay signé

\section{J. Nicolas Albert Couturier}

21. Sepult. de Catherine tginagarine. le dix a été Inhumé le corps de Catherine tginagarine sauvages du lac des montagnes, agée de deux ans, En foy de quoy j'ay signé

\section{J. Nicolas albert Couturier}

22. Ce dix neuvieme May de l'annee mil Sept cent Cinquante deux a été Batisé Jean $\mathrm{B}^{\text {te. }}$ né Le Jour de $\mathrm{l}^{\prime}$ ascension entre dix et onze heurs du matin fils de Joseph Guibeau et d'Angelique Berey ses pere et Mere en legitime mariage employés dans ce fort. Le Parain a été Sieur Mutigny de Vassan officier dans cette Garnison, Et la Maraine a été Demoiselle Elizabeth Lafavry Lesquels ont signé le present acte avec Nous 